Available online at: https://proceeding.researchsynergypress.com/index.php/cpmhs/index

RSF Conference Series: Medical and Health Science

e-ISSN (to be processed) / p-ISSN (to be processed)

Volume 1 Number 1 (2021): 106-111

\title{
Literature Review: Transplacental Transmission of COVID-19 and Its Teratological Aspect
}

\author{
Annisa Rahmah Furqaani' ${ }^{1}$, Arief Budi Yulianti ${ }^{1}$ \\ ${ }^{1}$ Public Medical Biology, Faculty of Medicine, Universitas Islam Bandung, Indonesia
}

\begin{abstract}
Coronavirus disease 2019 (Covid-19) pandemic affects all populations, including pregnant women. Severe acute respiratory syndrome coronavirus 2 (SARS-Cov-2) infection in pregnancy needs to be a concern because of the risk of transplacental transmission to the fetus and the potential to interfere with fetal development. The objective of this study is to review the transplacental transmission of COVID-19 and the teratological aspects of the event. This article is a literature study. Based on the literature obtained, placental infection, vertical transmission, and fetal infection have been identified in some cases. However, there is still no consistent and enough scientific evidence to show that those condition causes fetal damage or causes congenital anomalies. Virus and host characteristics are thought to explain why SARS-Cov-2 infection has not shown a teratological effect. SARS-CoV-2, similar to severe acute respiratory syndrome (SARS) and the Middle East respiratory syndrome (MERS) infection, does not indicate maternal-fetal transmission. The low-level expression of angiotensin-converting enzyme 2 (ACE2) and S protein priming proteases type II transmembrane serine protease (TMPRSS 2) in the placenta is also considered to be the factor that plays a role in inhibiting the vertical transmission of COVID-19. Adverse outcome of fetal death is more due to pathophysiological conditions of maternal health caused by SARS-CoV-2 infection during gestation.
\end{abstract}

Keywords: COVID-19, fetal development, teratology, transplacental transmission

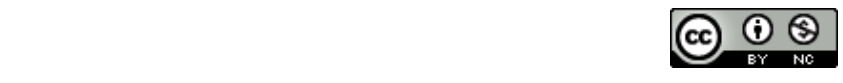

This is an open access article under the CC-BY-NC license

\section{INTRODUCTION}

The pandemic of COVID-19 has affected more than 200 million people worldwide, including pregnant women (WHO, 2021). Even though the effects of SARS-Cov-2 infection in pregnancy and fetal development remain relatively unclear, SARS-CoV-2 infection among pregnant women should become a major concern because of its potential risk of vertical transmission that may affect fetal development. The entry mechanism of SARS-CoV-2 into human cells is mediated by receptor ACE2. In addition, TMPRSS2 is also needed to facilitate the virus entry process. (Zhang \& Zhang, 2021; Pique-Regi et al., 2021; Hecht et al., 2020; Bohn et al., 2020). Studies reported that ACE2 is expressed by the placental cell, especially the syncytiotrophoblasts (Pique-Regi et al., 2021; Wong et al., 2021; Hecht et al., 2020; Hosier et al. 2020), that could potentially contribute to maternal-fetal transmission following COVID-19 infection during gestation. Many studies reported that SARS-Cov-2 infection throughout pregnancy increases the risk of complications, such as preeclampsia, intrauterine growth restriction, preterm birth, and low birth weight (Calvacante et al., 2021; Zhang \& Zhang, 2021; Hosier et al., 2020). To date, there is still no consistent evidence for the vertical transmission of SARS-CoV-2. Despite the placentas being confirmed infected by SARS-CoV-2, very few neonates manifest virus-induced signs and symptoms. Pique-Regi et al. (2020) reported that coincide expression of ACE2 and TMPRSS2 is insignificant in the placenta. In addition, Corresponding author Annisa Rahmah Furqaani, annisa.rahmah@unisba.ac.id; Arief Budi Yulianti, budi.yulifk@gmail.com DOI: (to be processed) 
minimal expression of ACE2 or TMPRSS2 protein by the human placenta throughout pregnancy could be the main factor that prevents congenital infections. The mechanisms underlying vertical transmission of SARS-CoV-2 are still poorly understood; its possibility of affecting fetal development or become a teratogenic agent is need to evaluate more. Broader, consistent, and scientific evidence to establish trophoblast SARS-CoV-2 infection and transplacental transmission is needed to conclude the teratogenic aspect of SARS-CoV-2.

\section{LITERATURE REVIEW}

The SARS-CoV-2 is a single-stranded RNA virus shrouded by envelopes classified as coronaviruses, especially $\beta$-coronaviruses. Apart from being enveloped by E protein, the surface of the SARS-CoV-2 virus consists of glycoproteins, known as S spike protein (S), membrane (M), and nucleocapsid (N). S protein plays a crucial responsibility in viral attachment and entry into host cells. The other glycoproteins (E, $M$, and $\mathrm{N}$ proteins) are involved in viral reproduction, particle assembly, and release from the host cells (Zare et al., 2021; Belmehdi et al., 2021; Park, 2020). SARS-CoV-2 needs to interact with the ACE2 receptor to enter cells, and in parallel, TMPRSS2 is required to $S$ glycoprotein breakage that allows viral fusion to the cell. Both ACE2 and TMPRSS2 are required to facilitate cell entry and spread of SARS-CoV-2 (Shang et al., 2020; Wang et al., 2020; Hoffmann et al., 2020). The existence of ACE2 on syncytiotrophoblasts cells of the placenta could potentially contribute to maternal-fetal transmission as the mother is diagnosed with positive COVID-19 during pregnancy. However, congenital infection or teratological effect of viral infection has led to three key points: (1) Identified/detected virus on the placenta tissue does not always convincing marker for placental infection; (2) placental infection does not always have to follow by vertical transplacental transmission; (3) If fetal infection detected, it is not always followed by fetal damage or caused congenital anomalies.

The placenta acts as an immunological barrier that selectively prevents pathogens' entry, in conjunction with its role in maintaining immune responses to the fetal development intrauterine. The innate immune system and structural barrier (outermost layer of chorionic villi is not equipped with intercellular gap junction) may contribute to the protective mechanisms of the placenta against viral invasion, including SARS-CoV-2 (Carsetti et al., 2020; Pereira, 2018). In addition, ACE2 and TMPRSS2 are poorly expressed by the human placenta in all trimesters of gestation so that SARS-CoV-2 tends not to infect the placenta and fetus (Wong et al., 2021; Hecht et al., 2020; Hoshier et al., 2020). Pique-Regi et al. (2020) also reported that the chorioamnionitis membranes do not express the ACE2 receptors in the third trimester. However, the severe maternal disease is associated with detected SARS-Cov-2's RNA in the amniotic fluid and newborn positivity at birth in some cases (Hosier et al., 2020; Vivanty et al., 2020; Zamaniyan, 2020). Although the villous syncytiotrophoblast provides an excellent barrier to pathogen infection, DNA viruses infection in the placenta has been widely known. As well as with RNA viruses are also reported to infect the placenta but often not causing histopathology changes. The placental's pathological characteristics exposed to SARS-CoV-2 have not been demonstrated clearly. However, coagulopathic findings shared with features such as Hofbauer cell hyperplasia, chronic histiocytic intervillositis, or massive perivillous fibrin deposition, often described as the histopathology appearance of placenta's patients with SARS-CoV-2 infection (Bergen \& Heller, 2020; Shuo et al., 2020; Shanes et al., 2020).

Some studies found that the expression of ACE2 in placenta and decidua cells is generally very lowlevel (Zheng et al., 2020; Vento-Tormo et al., 2018). Pique-Regi et al. (2020) observed insignificant colocalization of ACE2 and TMPRSS2 in the placenta and fetal membranes in the third trimester of 
pregnancy. On the other hand, some studies showed that ACE2 and TMPRSS2 are expressed in the trophoblast, the blastocyst, and the hypoblast in the first trimester of pregnancy, so fetal infection is possible through this pathway in the early gestation periods (Weatherbee et al., 2020; Zheng et al., 2020). However, there is still no established evidence, and rare events show the impact of COVID-19 in early gestation (Wastnedge et al., 2021). The lack of coexpression of ACE2 and TMPRSS2 in the placenta leads to the alternative mechanism that SARS-CoV-2 possibly prefers to enter the placental tissues (Kreis et al., 2020; Pique-Regi et al., 2020). Several proteases are proposed to be involved in SARS-CoV-2 entry to the cell, such as dipeptidyl peptidase-4 (DPP4) and the cluster of differentiation 147 (CD147), both of which are highly expressed in the placenta during pregnancy (Kreis et al., 2020). Like TMPRSS2, cathepsins B and $\mathrm{L}$, trypsin, and furin have been studied to affect $\mathrm{S}$ glycoprotein's conformation so that it allows the virus to access S1/S2 binding site (Hoffman et al. 2020; Hu et al., 2020; Pique-Regi et al., 2020). Therefore, it is still very possible that fetal infection of SARS-CoV-2 occurs and interferes with fetal development.

\section{RESEARCH METHODOLOGY}

This article is a literature review. Articles were obtained by doing a search on the databases: Google Scholar, Pubmed, and Science Direct. Keywords used in the search: COVID-19, vertical/transplacental transmission, and fetal development/congenital infection/teratology. The authors then reviewed the appropriate literature to study the mechanism of vertical transmission of COVID-19 and its potency in affecting fetal development, causing congenital abnormalities.

\section{FINDINGS AND DISCUSSION}

All reviewed articles have explained that transplacental transmission of SARS-Cov-2 has been detected but not always followed by fetal infection. In very rare cases, the fetal infection has been observed but has not been associated with teratological aspects or caused congenital abnormalities in the fetus. However, COVID-19 infection during gestation may have implications for both the mother and fetus's health. Pregnant women with COVID-19 can show ranging from mild to severe symptoms. Poor maternal health can affect fetal development; the disruption of fetal development is not due to a viral infection but rather due to the mother's declining health condition during pregnancy. Maternal vascular malperfusion and inflammatory changes are observed in pregnant women infected with even mild COVID-19 (Mahyuddin et al., 2020). COVID-19 infection in pregnancy correlates with increased risks of adverse pregnancy outcomes, such as intrauterine growth restriction, preterm birth, low birth weight, and perinatal mortality (Wastnedge et al., 2021; Cavalcante et al., 2021). Those conditions have been associated with non-communicable diseases in neonates, such as neurological and behavioral disorders. Maternal immune responses to viral infection, including COVID-19, are responsible for the offspring's possible risk rather than the virus itself. Mulvey et al. (2020) reported histology observation of five COVID19 patients, indicating no vascular complement deposition in the placentas. Nevertheless, fetal vascular malperfusion was observed, characterized by focal avascular villi and thrombi in larger fetal vessels. The study also showed negative results of viral RNA fraction and S glycoprotein determination. All patients experienced healthy and term deliveries; these findings suggest that COVID-19 has been involved in procoagulant activity, but it is unrelated to systemic complement activation. Vivanty et al. (2020) demonstrated that 23-year-old women, gravida 1, para 0, infected by SARS-CoV-2 (35 weeks gestation), cause maternal viremia, placental infection and inflammation, neonatal viremia following placental infection. The neonate presented with neurological manifestations. Correspondingly, changes in the maternal immune response during pregnancy and COVID-19 infection are thought to be associated with 
possible transplacental transmission of SARS-CoV-2 and neurotropism that may affect the fetus's neurodevelopment risk of psychosis, schizophrenia, and schizophrenia spectrum disorders (Zimmer et al., 2021). Schwartz (2020) reported that 29 newborns delivered by mothers diagnosed with positive COVID19 showed negative RT-PCR of SARS-CoV-2 and negative SARS-CoV-2 of their placenta. To date, the behavior of vertical transplacental transmission of SARS-CoV-2 in pregnancy is similar to other coronavirus infections (SARS and MERS), which have not been conclusively proven (Schwartz \& Dhaliwal, 2020; Chen et al., 2020).

Although maternal-fetal transmission of SARS-CoV-2 is rare, Zheng et al. (2020) reported that a small fraction of the virus was detected in neonates at 1-4 days after birth but then reported negative on days 6-7. The serological examination also revealed that detectable increases in neonatal immunoglobulin (Ig)M and IgG were allied with maternal confirmed with COVID-19 (Zeng et al., 2020; Dong et al., 2020). Transplacental transfer of IgG is suggested to be a mechanism for this immunoglobulin enhancement. In contrast, elevated levels of IgM are stimulated by fetal SARS-CoV-2 infection. Furthermore, the studies reported that all neonates did not manifest any symptoms related to COVID-19 (Zeng et al., 2020; Dong et al., 2020). Severe COVID-19 affected mother showed detectable viral RNA in amniotic fluid and delivered premature neonates that showed positive results after 24 hours delivery (Zamaniyan, 2020). The study of Hosier et al. (2020) reported that all fetal tissues tested are negative SARS-CoV-2, in contrast to positive results of viral RNA in the placenta and umbilical cord of the mothers. The mothers reported having severe preeclampsia, placental abruption, or other complications during pregnancy. Pique-Regi et al. (2020) found low levels co-express ACE2 and TMPRSS2 by the placental cells throughout gestation. The firsttrimester data showed low-level expression of ACE2 at the human placenta. Insignificant coexpression of ACE2 receptors and TMPRSS2 was also observed in the chorioamnionitis membranes in the third trimester. Therefore, Pique-Regi et al. (2020) suggested that other concomitant pathological conditions involved facilitating vertical transmission of SARS-CoV-2, resulting in a breach of the maternal-fetal crosstalk. To date, the data indicate that the characteristics of SARS-CoV-2 make the virus less likely to infect human placental, so that minimize the transplacental transmission. However, some pathological conditions in pregnancy strongly suggest taking responsibility for increasing the expression of ACE2 (Alexandre et al., 2020) by altering the renin-angiotensin-aldosterone system (RAAS). In addition, high levels of DPP4 and CD147 are expressed in the placenta during pregnancy, so that potentially used by the SARS-CoV-2 to enter the placental cells; replace the role of TMPRSS2 (Kreis et al., 2020). The changes in viral S glycoprotein conformation could also be facilitated by cathepsins B and L, trypsin, and furin, allowing viral attachment at the S1/S2 binding site (Hoffman et al. 2020; Hu et al., 2020; Pique-Regi et al., 2020). Accessible cellular receptors, entry pathways, and invasive mechanisms that could be developed by SARS-CoV-2, especially in pregnancy and fetus, are still under investigation. To evaluate vertical transmissions and their possibility of causing congenital anomalies, broader and longer-term observations are required so that a comprehensive understanding of the teratological aspect of SARS-CoV-2 could be established.

\section{CONCLUSION AND FURTHER RESEARCH}

It can be concluded that the vertical transmission of COVID-19 has been observed, although it is still rare, and the mechanism of transmission is not clearly established. However, this event is not always followed by fetal infection, so that the teratological aspects of COVID-19 have not been identified yet. More significant data and long-term observation are needed to evaluate the effects of congenital infection of SARS-CoV-2 on the fetus. 


\section{REFERENCES}

Baergen RN, Heller DS. (2020). Placental pathology in Covid-19 positive mothers: preliminary findings. Pediatr Dev Pathol, 23, pp. 177-80.

Belmehdi O, Hakkour M, El Omari N, Balahbib A, Guaoguaou FE, Benali T, et al. (2021). Molecular structure, pathophysiology, and diagnosis of COVID-19. Biointerface Research in Applied Chemistry, 11, 3, 10215-37.

Bohn MK, Hall A, Sepiashvili L, Jung B, Steele S, Adeli K. (2020). Pathophysiology of COVID19: Mechanisms underlying disease severity and progression. Physiology, 35, 288-301.

Carsetti R, Quintarelli C, Quinti I, Piano Mortari E, Zumla A, Ippolito G, Locatelli F. (2020). The immune system of children: The key to understanding SARS-CoV-2 susceptibility? Lancet Child Adolesc. Health, 4, 414-16.

Cavalcante MB, Cavalcante CTMB, Sarno M, Barini R, Kwak-Kim J. (2021). Maternal immune responses and obstetrical outcomes of pregnant women with COVID-19 and possible health risks of offspring. Journal of Reproductive Immunology,143, https://doi.org/10.1016/j.jri.2020.103250.

Chen H, Guo J, Wang C, Luo F, Yu X, Zhang W, et al. (2020). Clinical characteristics and intrauterine vertical transmission potential of COVID-19 infection in nine pregnant women: a retrospective review of medical records. Lancet, 395, 809-15.

Dong L, Tian J, He S, Zhu C, Wang J, Liu C, Yang J. (2020). Possible vertical transmission of SARS-CoV-2 from an infected mother to her newborn. Jama, 323, 1846-1848. DOI: https://doi.org/10.1001/jama.2020.4621

Hecht JL, Quade B, Deshpande V, Mino-Kenudson M, Ting DT, Desai N, et al. (2020). SARSCoV-2 can infect the placenta and is not associated with specific placental histopathology: a series of 19 placentas from COVID-19-positive mothers. Modern Pathology, 33, 2092-103.

Hoffmann M, Kleine-Weber H, Schroeder S, Krüger N, Herrler T, Erichsen S, et al. (2020). SARS-CoV-2 cell entry depends on ACE2 and TMPRSS2 and is blocked by a clinically proven protease inhibitor. Cell, 181, 271-80. DOI: https://doi.org/10.1016/j.cell.2020.02.052, PMID: 32142651

Hosier H, Farhadian SF, Morotti RA, Deshmukh U, Lu-Culligan A, Campbell KH, et al. (2020). SARS-CoV-2 infection of the placenta. J Clin Invest, 130, 9, 4947-53. https://doi.org/10.1172/JCI139569.

https://covid19.who.int/ accessed 12th August 2021.

Kreis NN, Ritter A, Louwen F, Yuan J. (2020). A message from the human placenta: structural and immunomodulatory defense against SARS-CoV-2. Cells, 9, 8, 1777. doi:10.3390/cells9081777

Mahyuddin AP, Kanneganti A, Wong JJL, Dimri PS, Su LL, Biswas A, et al. (2020). Mechanisms and evidence of vertical transmission of infections in pregnancy, including SARS-CoV-2s. Prenatal Diagnosis, 4, 1655-70.

Mulvey JJ, Magro CM, Ma LX, Nuovo GJ, \& Baergen RN. (2020). Analysis of complement deposition and viral RNA in placentas of COVID-19 patients. Annals of Diagnostic Pathology, 46. https://doi.org/10.1016/j.anndiagpath.2020.151530

Park SE. (2020). Epidemiology, virology, and clinical features of severe acute respiratory syndrome -coronavirus-2 (SARS-CoV-2; Coronavirus Disease-19). CEP, 63, 4, 119-24. https://doi.org/10.3345/cep.2020.00493

Pereira, L. (2018). Congenital viral infection: Traversing the uterine-placental interface. Annu. Rev. Virol., 2018, 5, 273-299. 
Pique-Regi R, Romero R, Tarca AL, Luca F, Xu Y, Alazizi A, et al. (2020). Does the human placenta express the canonical cell entry mediators for SARS-CoV-2? eLife, 9, e58716. DOI: https://doi.org/10.7554/eLife.58716

Schwartz DA \& Dhaliwal A. (2020). Infections in pregnancy with COVID-19 and other respiratory RNA virus diseases are rarely if ever, transmitted to the fetus. Arch Pathol Lab Med, 144, 920-28. DOI: 10.5858/arpa.2020-0211-SA

Schwartz DA. (2020). An analysis of 38 pregnant women with COVID-19, their newborn infants, and maternal-fetal transmission of SARS-CoV-2. Arch Pathol Lab Med., 144, 799-805. DOI: 10.5858a/arpa.2020-0901-SA)

Shanes ED, Mithal LB, Otero S, Azad HA, Miller ES, Goldstein JA. (2020). Placental pathology in COVID-19. Am J Clin Pathol., 154, 23-32.

Shang J, Ye G, Shi K, Wan Y, Luo C, et al. (2020). Structural basis of receptor recognition by SARS-CoV-2. Nature, 581, 221-24. DOI: https://doi.org/10.1038/s41586-020-2179-y

Shuo C, Bo H, Danju L, Xiang L, Fan Y, Yin Z, et al. (2020). Clinical characteristics and pathological analysis of placenta in three cases of pregnant women with novel coronavirus infection. Chin J Pathol., 49, 418-23.

Vento-Tormo R, Efremova M, Botting RA, Turco MY, Vento-Tormo M, Meyer KB, et al. (2018). Single-cell reconstruction of the early maternal-fetal interface in humans. Nature, 563, 347-53. DOI: https://doi.org/10.1038/s41586-018-0698-6, PMID: 30429548

Vivanti AJ, Vauloup-Fellous C, Prevot S, Zupan V, Suffee C, Cao JD, et al. (2020). Transplacental transmission of SARS-CoV-2 infection. Nature Communications, 11, 3572. https://doi.org/10.1038/s41467-020-17436-6.

Wang Q, Zhang Y, Wu L, Niu S, Song C, Zhang Z, et al. (2020). Structural and functional basis of SARS-CoV-2 entry by using human ACE2. Cell, 181, 894-04. DOI: https://doi.org/10.1016/j.cell.2020.03.045, PMID: 32275855

Weatherbee BAT, Glover DM, \& Zernicka-Goetz M. (2020). Expression of SARS-CoV-2 receptor ACE2 and the protease TMPRSS2 suggests susceptibility of the human embryo in the first trimester. Open Biol, 10, 200162. doi:10.1098/rsob.200162.

Wong YP, Khong TY, \& Tan GC. (2021). The effects of COVID-19 on placenta and pregnancy: what do we know so far? Diagnostics, 11, 94, 1-13. https://doi.org/10.3390/diagnostics11010094

Zamaniyan M. (2020). Preterm delivery in pregnant woman with critical COVID-19 pneumonia and vertical transmission. Prenatal Diagnosis, 7, 5713. DOI: https://doi.org/10.1002/pd.5713

Zare A, Sadati-Seyyed-Mahalle SF, Mokhtari A, Pakdel N, Hamidi Z, Almasi-Turk S, et al. 2021. An update of coronavirus disease 2019 (COVID-19): an essential brief. Mod Med Lab J., 4, 1, 19-38.

Zhang H \& Zhang H. (2021). Entry, egress and vertical transmission of SARS-CoV-2. Journal of Molecular Cell Biology, 1, 3, 168-74.

Zheng Q-L, Duan T, \& Jin L-P. (2020). Single-cell RNA expression profiling of ACE2 and AXL in the human maternal-Fetal interface. Reprod Dev Med., 4, 7. doi:10.4103/2096-2924.278679

Zimmer A, Youngblood A, Adnane A, Miller BJ, Goldsmith DR. (2021). Prenatal exposure to viral infection and neuropsychiatric disorders in offspring: A review of the literature and recommendations for the COVID-19 pandemic. Brain, Behavior, and Immunity, 91, 756-70. https://doi.org/10.1016/j.bbi.2020.10.024 